\title{
Corrective surgery for Parkinson's disease (PD) deformity following Deep Brain Stimulation (DBS) - Is DBS a last resort?
}

\author{
Jun Mizutani ${ }^{1 *}$, Muneyoshi Fukuoka', Nobuyuki Suzuki', Seiji Otsuka', Atsushi Umemura², Yuichi Oka², \\ Kazuo Yamada ${ }^{2}$, Takanobu Otsuka ${ }^{1}$ \\ From The 10th Meeting of the International Research Society of Spinal Deformities (IRSSD 2014 Sapporo) \\ Sapporo, Japan. 29 June - 2 July 2014
}

\section{Objective}

Spinal deformity related to Parkinson's disease (PD) is severe. Due to reported high complication rate, some authors reported that PD deformity with no neurological symptoms should not undergo surgery and also reported deep brain stimulation (DBS) should be considered instead of corrective surgery for their postural disorders. However, almost of previous reports did not consider spino-pelvic parameters. If spino-pelvic harmonization is achieved even in PD deformity surgery, there is a possibility of obtaining much better surgical results. We analyzed the surgical results of PD deformity surgery focused in spino-pelvic parameters and we here report minimum two years follow up of PD deformity surgery.

\section{Patients and methods}

Six patients with Parkinson's deformity following DBS underwent corrective surgery were enrolled this study. Mean age at surgery was 67.8 y.o. and there were 3 men and 3 women. Pelvic incidence, Pelvic tilt, SVA, lumbar lordosis, thoracic lordosis, JOA score were analyzed pre-, and post-surgery.

\section{Results}

Five patients whose SVA was over $100 \mathrm{~mm}$ underwent thoracic to sacro-iliac fixation. Remaining one patient whose SVA less than $100 \mathrm{~mm}$ underwent L2-5 PLIF only. Mean SVA in long fixed patients was improved less than $100 \mathrm{~mm}$. Lumbar lordosis in long fusion patient was improved to 30-50 degrees from flatback.
Short fusion patient's alignment following surgery was maintained. SVA was $55 \mathrm{~mm}$ and lumbar lordosis was 47 degrees.

\section{Conclusion}

Although many literature have reported early breakage or complications may occur in spinal surgery in Parkinson's patients, when the PD deformity surgery is planned, considering into spino-pelvic harmonization, PD deformity spine surgery might be reduced their symptoms and the alignment might be maintained well in spite of the fusion level.

\section{Authors' details \\ 'Department of Orthopaedic Surgery, Nagoya City University, Japan. \\ ${ }^{2}$ Department of Neurosurgery, Nagoya City University, Japan.}

Published: 19 January 2015

doi:10.1186/1748-7161-10-S1-O55

Cite this article as: Mizutani et al.: Corrective surgery for Parkinson's

disease (PD) deformity following Deep Brain Stimulation (DBS) - Is DBS a last resort? Scoliosis 2015 10(Suppl 1):O55.

${ }^{1}$ Department of Orthopaedic Surgery, Nagoya City University, Japan

Full list of author information is available at the end of the article 